\title{
Über Priapismus.
}

Von

\author{
Dr. J. von Broich, \\ Spezialarz für Hautkrankbeiten in Elberfeld.
}

Unter Priapismus oder Satyriasis verstehen wir eine übermäBig starke Erektion des Penis, die sich von der normalen durch das Fehlen jeden Wollustgefühles unterscheidet und entweder nur anfallsweise auftritt, meistens jedoch andauert und sich auf Tage und Wochen erstrecken kann.

Am 10. Juli 1899 wurde ich zu einem Kranken gerufen, der seit ca. 20 Stunden an ununterbrochener Frektion des Penis litt, begleitet von unerträglichen Schmerzen. Schon vor 8 Tagen hatte. Pat. schon einmal das zweifelhafte Vergnügen gehabt, 2-3 Stunden lang seinen Penis andauernd erigiert zu sehen. Die Erektion ging damals bald unter kalten Umsehlägen zurück, ohne irgendwelche Nachwirkungen zu hinterlassen. Seit gestern Abend hatte erneut eine heftige Erektion begonnea, zu deren Beseitigung Pat. zunäehst einen 20 Minuten danernden Coitus versucht hatte, jedoch obne Erfolg und ohne einen Samenerguß erreichen zu können. Kalte Umschläge und Eisbeutel blieben auch ohne Wirkung.

Ioh suchte mir nun zunächst ans der Anamnese die Ursache zu ergründen, wodurch diese andauernde Erektion hervorgerufen sein könnte.

Pat, 31 J. alt, kam zuerst in meine Behandlung am 9./IV. 1896 wegen Lues (also vor 3 Jahren,) die er $1^{3 / 4}$ Jahre früher (Mitte 1894) akquiriert hatte und gegen die er bereits 2 Schmierkuren durchgemacht hatte. Es bestanden damals Plaqu. muqueuses tonsill., Defluv. cap. syph. Einen Monat später erkrankte seine Frau, die im 5. Mon. gravida war, auch an Lues (Roseola, Condyl. lat., Plaqu. muqu.) Die angeordnete Schmierkur wurde von beiden Ehegatten dann in Aachen durchgeführt. Die Frau gebar damals ein totes Kind; ein späteres zweites Kind wurde jedoch ausgetragen, war und blieb gesund, ohne jemals syphilitische Symptome zu zeigen. 
An Gonorrhoe hatte Pat. vor seiner Ehe wiederholt gelitten. Nach mehrjähriger Pause hatte er sich dann jetzt wieder vor ca. $2^{1 / 2}$ Monaten gonorrhoisch infiziert, hatte jedoch nach seiner Angabe sich die Gonorrhoe mit einem vorzüglichen Mittel selbst sehr schnell kuriert, und habe er schon seit mehreren Wochen nicht die geringste Spur von Tripper mehr wahrnehmen können. Für die Heilung sprach dann auch der Umstand, dafs er seine Frau nicht gonorrhoisch infiziert hatte.

In den letzten Wochen hatte er nach Aussage seiner Frau außerdem stark dem Alkoholgenuß gefrönt, er habe sehr viel und bäufig bis tief in die Nacht hinein gezecht und sei sehr häufig früh Morgens bekneipt nach Hause gekommen. Aufregungen im Geschäft taten das übrige, ihn höchst nervös zu machen, so daß er zeitweise über die geringsten Kleinigkeiten außerordentlich erregt und heftig wurde.

Somit waren 3 Momente gegeben, die bei der Beurteilung der Erkrankung als ursächliches Moment in Frage kommen konnten: Lues, Gonorrhoe und Alkoholismus.

Die Untersuchung ergab nun folgendes:

Der Penis ist sehr kräftig erigiert, mit Ausnahme der Glans, die von gewöhnlicher, normaler Größe ist und vom Präputium bedeckt wird; sie ist demgemäß nicht sichtbar. Der Penis ist stark nach dem Bauch aufwärts geneigt und reicht fast bis zum Nabel. Der Winkel zwischen Penis und Bauchdecken beträgt knapp $45^{\circ}$. Eine Bewegung des Penis nach oben oder wester nach unten zum Bauche hin, nach rechts oder links, ist unmöglich. Die Schwellkörper fühlen sich hart an, sind strotzend gespannt, an ihrer Wurzel ca. $6 \mathrm{~cm}$ breit. Arterienpulsation ist an verschiedenen Stellen zu fühlen, die Hautvenen sind stark gefüllt. Die Haut des Penis ist sonst unverändert, ohne Zeichen von Entzündung, ohne jegliche Verfärbung. Das Präputium läBt sich leicht zurückschieben, die Urethralöffnung ist nicht geschwollen, nicht gerötet, nicht verklebt, nichts Besonderes läßt sich an derselben feststellen, auch auf Druck läBt sich kein Sekret aus der Urethra hervorbringen. Eine genaue Betastung des erigierten Penis auf irgendwelche besondere Anschwellung oder KnotenbiIdung ergibt ein völlig negatives Resultat, obschon die Untersuchung trotz der allenthalben bei Berührung vorhandenen heftigen Schmerzen ziemlich schonungslos durchgeführt wurde. Es folgte dann die Untersuchung per rectum; die dem Patienten sehr schmerzhafte Untersuchung förderte auch keine weiteren Anhaltspunkte zu Tage, die Prostata ist absolut nicht geschwollen, desgl. die Samenblasen nicht, überhaupt sind alle Organe hier, soweit sie mit dem Finger abgetastet werden können, völlig normal, insbesondere ist keine Spur eines Zirkulationshindernisses, etwa infolge eines in Bildung begriffenen Abszesses nachweisbar. Der Urin, ohne 
Beschwerden in Knieellbogenlage entleert, ist klar, beim Stehen setzt sich nur eine ganz leichte Wolke ab, Fäden sind nicht vorhanden, desgl. weder Zucker noch Eiweiß. Kein Fieber. Sensorium frei. Die Sehnenreflexe sind gesteigert. Im übrigen fühlt sich Pat. wohl, klagt nur über die unerträglichen Schmerzen der fortdauernden Erektion im Penis. Irgendwelche Symptome von Syphilis sind nicht nachweisbar. Alle übrigen Organe sind gesund, speziell ist keine Milzschwellung vorhanden.

Therapeutisch wurden zunächst Narcotica verordnet, wobei sich nach einer Morphiuminjektion von $0^{\circ} 02$ das interessante $\mathrm{Phänomen} \mathrm{zeigte,}$ $\mathrm{da} B$ an der Injektionsstelle am linken Unterarm sich unter heftigem Jucken innerhalb einer Minute eine talergroße Urticariaquaddel bildete.

Da die Untersuchung auf frische Symptome von Lues und Gonorrhoe negativ ausgetallen war, so neigte ich mehr zu der Ansicht, daß es sich um Folgen des Alkoholmißbrauches handle, und daß der Priapismus erklärt werde durch eine Nervenreizung. Von dieser Erwägung ausgehend und gleichzeitig, um eine noch eingehendere örtliche Untersuchung zo ermöglichen, beschloB ich am folgenden Tage, unter Assistenz des Hausarztes, den Patienten zu chloroformieren, nachdem er trotz reichlichen Nareoticis eine sehr schlechte Nacht gehabt hatte. Die Narkose bestätigte die anamnestischen Angaben des Potatoriums, Pat. war sehr unruhig und mubte sehr tief chloroformiert werden. Die therapeutische Wirkung der Narkose auf den erigierten Penis war jedoch gleich Null. Eine uochmalige genaue Abtastung auf etwa vorhandene Infiltrate im Penis, Ausquetschung der Urethra auf vorhandenes Sekret, genaue Untersuchung per rectum auf Veränderungen in der Prostata oder in den Samenblasen, Dehnung des sphincter ani, blieben resultatlos.

Örtlich Eisblase, innerlich Brom in großen Dosen brachten dem Patienten etwas Ruhe. Am folgenden Tage mußte jedoch Brom wieder ausgesetzt werden, da Pat. zu viel genommen hatte und sich Vergiftungssymptome bemerkbar machten, zunächst durch heftige Kopfschmerzen später durch stark ikterische Hautfärbung. Ein auf Anordnung des Hausarztes gegebenes kaltes Bad von $12^{\circ} \mathrm{R}$. war dem Pat. außerdem nach 2 Min. Dauer schon so schlecht bekommen, daß sich Kollapserscheinungen und Erbrechen einstellten. Die Harnentleerıng sistierte, and wurde die Einführung des Katheters notwendig, die sehr leicht von statten ging und $3 /, l$ tiefdunkeln, jedoeh klaren Harn zu Tage förderte. Die Schmerzen im Penis waren jetzt so stark geworden, daß Pat. nicht mehr aufstehen und sich nicht mehr auf die Seite legen konnte, sondern nnentwegt die Rückenlage einhalten mußte. Außerdem klagte er über heftiges und unerträgliches Ziehen in den Hoden. Die Urinentleerung erfolgte bald jedoch wieder spontan, und die Behandlung beschränkte sich auf ein zweimal tägliches, temperiertes $\mathrm{Bad}$ von $25^{\circ} \mathrm{R}$. mit $\mathrm{Ab}$ kühlung bis auf $22^{\circ}$ innerhalb 5 Minuten, und auf die Darreichung von Narcotica (Trional 1.0 + Morph. mur. 0.015). 
Unterdessen war unter der fünftägigen Eisbehandlung die Haut des Penis blaurot geworden, die Schmerzen waren unverändert, so daß jetzt warme Kataplasmen angewandt wurden, die denn auch dem Patienten etwas Linderung brachten.

Zur Entscheidung, ob es sich um eine Nervenreizung handle oder nicht, machte ich dann einem noch zugezogenen Nervenarzte den Vorschlag, den Patienten zu hypnotisieren. Die Hypnose gelang wider Erwarten sehr gut, Pat. schlief fest etwa 15 Minuten, mußte aber zu seiner und unserer Enttäuschung nach dem Aufwachen wahrnehmen, daß die Erektion vor wie nach unverändert geblieben war.

Somit kamen wir zu der Überzeugung, daß es sich um eine Thrombose in den corpora cavernosa penis handle, deren Ursache uns vorläufig unbekannt war.

Nach 11tägigem Bestehen der Erektion zogen wir dann als Consiliarius noch Herrn Geheimrat Doutrelepont zu, der nochmals eine sehr genaue Rektaluntersuchung vornahm, ohne etwas abnormes dort feststellen zu können, jedoch die Vermutung aussprach, es könne sich um eine gonorrhoische Erkrankung handeln, und demgemäß zunächst warme Borwassereinspritzungen in die Urethra verordnete und für später Instillationen in die Pars. post. urethrae anriet. - Direkt nach der ersten Einspritzung, die ich am folgenden Tage selbst gemacht hatte, zeigte sich unmittelbar nach dem Ablaufen der $3 \%$ Borlösung merkwürdigerweise ein Tropfen Eiter, der zahlreiche Gonokokken enthielt, während bis dato alle Untersuchungen auf Gonorrhoe negativ ausgefallen waren. Es war trotz wiederholter Untersuchung, trotzdem Pat. auf Geheiß selbst stets darauf achtete, nie eine Spur Sekret gesehen worden, und merkwürdigerweise war auch der Urin stets frei von Sedimenten und Filamenten gewesen.

Selbstverständlich ging die Direktive in der weiteren Behandlung darauf aus, den gonorrhoischen Prozeß zu bekämpfen, um so mehr, als schon am folgenden Tage die nunmehr offenbar frei gewordenen Gonokokken eine heftige Cystitis hervorgerufen hatten, die sofortige Blasenausspülungen erheischte. Ich bemerke dabei, daßj die Einführung des weichen Nelatonkatheters außerordentlich leicht vor sich ging, and dab die Spülungen, obschon nur ein gelindes Mittel angewandt wurde, nämlich 3prozentige Borsäurelösung bald den gewünschten Erfolg hatten.

Ich trat dann am 25. Juli meine Urlaubsreise an, und hatte Herr Kollege Strauss aus Barmen dann die Liebenswürdigkeit, den Pat weiter zu behandeln und mir über den weiteren Verlauf zu berichten.

Außer den Blasenspülungen wurden dann noch Instillationen in die pars. post. urethrae mit 5\% Argoninlösung, später mit Protargol $2 \%$ gemacht, die Harnbeschwerden bekämpft mit narkotischen Suppositorien, und protahierte warme Bäder bis za 4 Stunden Dauer verwandt, um die 
Resorption der Thrombose im Penis zu beschleunigen. Einige Male an den Penis angesetzte Blutegel blieben trotz heftiger Blutung ohne Erfolg. Eine besondere Erwähnung verdient noch der Umstand, daß zuweilen nach dem Katheterismus, resp. nach der Blasenspülung beim Herausziehen des Katheters aus der Urethra ein Tropfen Fiter erschien, und ebenso, daß zeitweise nach Schluß der Miktion ein eitriges Sekret sich zeigte, das den schon klaren Urin in seiner letzten Portion trübte. Die Untersuchung dieses im Spitzglase aufgefangenen Sekretes ergab Eiter mit zahlreichen, meist extrazellulär gelagerten Gonokokken (n. Gram). Der Ausfluß aus der Urethra vor der Harnentleerung dagegen war meist schleimiger Art und nach 8tägiger Behandlung schon frei von Gonokokken. Die Schmerzen im Penis waren allmählich unter der Behandlung mit Priessnitzschen und warmen Breiumschlägen erträglicher geworden. Zur Vereinfachung dieser Behandlung wurden dann aus Aluminium hergestellte Kapillarschlingen um den Penis gelegt, durch die beständig lauwarmes Wasser zirkulierte.

Endlich am 7./VIII., also nach 29 Tagen seit Beginn der Erektion begann der Penis deutlich zu fallen. Die Konsistenz wurde weicher jedoch ungleichmäßig, links mehr wie rechts, so daß der Penis sich nach links hinüber legte; namentlich am Bulbus rechts blieb die Härte noch ziemlich fest. Pat. konnte jedoch schon einige Stunden außer Bett zubringen. Die Resorption ging dann langsam weiter, anfangs konnte rechts im corp. cavernos. immer noch eine strangförmige Verhärtung konstatiert werden.

Nach einem halben Jahre sah ich den Patienten wieder. Er gab an, sich körperlich völlig wohl zu fühlen, er klagte nur darüber, daß die Erektionen nicht so kräftig seien wie früher, das Membrum biege sich beim Einführen in die Vagina hin und her und die Ejakulation erfolge ihm zu schnell. Nach der ersten Pollution, die 8 Tage nach dem Aufhören des Priapismus erfolgte, seien die Pollutionen anfangs während seiner Erholungsreise sehr häufig gewesen, fast jeden zweiten Tag. Die Gonorrhoe war geheilt. Die Syphilis machte sich jedoch später noch einmal bemerkbar. Am 10./VI. des folgenden Jahres (1900) konnte ich ein papulöses Infiltrat am Skrotum konstatieren, weswegen jch dem Pai. eine Injektion von Hydrarg. sal. machte. Pat. blieb dann aus und hat, wie ich später von seiner Frau hörte, nochmals eine Sehmierkur gemacht.

In diesem höchst interessanten Falle hatte sich also die Gonorrhoe als Ursache des Priapismus herausgestellt, eine Tatsache, die, soweit mir die Literatur über Priapismus bekannt geworden ist, bis jetzt nur in 2 Fällen [Soresin $\left.a^{1}\right)$ ] mitgeteilt ist. Nimmt man die verschiedensten Lehrbücher über Geschlechtskrankheiten zur Hand, so findet man meistens nicht einmal den Namen Priapismus verzeichnet; findet man

1) Arch. f. Derm. 1869. I. 603. 
aber im Register das Wort "Priapismus", so erhält man eventuell die Definition, daß es eine schmerzhafte, andauernde Erektion ohne Wollustgefühl sei, und weiter nichts. In anderen Lehrbüchern z. B. über Pathologie oder über Nervenkrankheiten finden sich meist nur ganz kurze Angaben. Glücklicherweise scheint also die Erkrankung eine ausnahmsweise seltene zu sein. Einzelne Fälle finden sich angedeutet in Eulenburgs Realenzyklopädie ${ }^{1}$ ) unter "Penis“. Demarquay und Bar lemont (20 Tage) beobachteten Priapismus nach einer Schußverletzung, Smith bei einem Potator nach Fall auf das Mittelfleisch, 5 Wochen, Hird bei einem Potator 6 Wochen. Clary einen Fall von 2 Monaten Dauer. In 2 Fällen von Maekel und Birket kam es nach Schüttelfrösten zur Eiterung, Thaut behandelte einen nur anfallsweise auftretenden Pria. pismus. Etwas ausführlicher beschriebene Fälle fand ich in einer Dissertation von $\mathrm{Adams}^{2}$ ) aus der Bonner med. Klinik (Prof. Schultze). Adams berichtet über einen Fall von Leukämie mit Priapismus. Der Kranke litt an starker Milzschwellung, Vermehrung der weißen Blutkörperchen und an einem Priapismus, der im ganzen 6 Wochen anhielt. In der Literatur konnte A. 5 Fälle auffinden, in denen Leukämie mit Priapismus verbunden war. Klemmé, ${ }^{3}$ ) Louquet, ${ }^{4}$ ) N eidhart, ${ }^{5}$ ) Matthias, ${ }^{6}$ ) Salzer, ${ }^{7}$ ) berichten solche Fälle, in denen die Leukämie die Ursache der Erkrankung war, bei allen war eine bedeutende Milzvergrößerung zu konstatieren. Schultze sieht als Ursache des "Priapismus leukaemicus" die Thrombenbildung an. Die veränderte Blutbeschaffenheit durch die Vermehrung der weißen Blutkörperchen führt zu einer Verlangsamung des Blutstromes, zu Abscheidungen und - Verklebungen von weißen Blutkörperchen an den Gefäßwandungen des kavernösen Gewebes. Es kommt zu Stauungen im Venengebiet und zur Bildung der sog. weißen Thrombose. Die Folge davon ist höchste Schwellung der corp. cavernosa, außerordentliche Spannung und Schmerzhaftigkeit. Salzer glaubt in seinem Falle den Priapismus auf Nervenreizung zurückführen zu müssen, auf einen abnorm lang dauernden Reizungszustand der nervi erigentes, während Lou qu e t annimmt, daß in seinem Falle außer der Thrombosenbildung wahrscheinlich

1) 3. Auflage 1898.

2) Dissert. Bonn. 1891 .

3) Inaug.-Dissert. Cassel. 1863.

4) Progés médic. 32. 1876.

5) Allg. med. Zentral-Ztg. 55. 1876.

6) Allg. med. Zentral-Ztg. 97 und 98. 1876.

7) Berl. klin. Wochenschr. 11. 1879. 
noch Hämorrhagien, wie solche auch aus Nase und Rektum bestanden, hinzugekommen seien.

Bei der pbysiologischen Erektion haben wir bekanntlich infolge Gefäßdilatation einen stärkeren arteriellen Blutzufluß in die Maschenräume der corpora cavernosa durch Reizung der nervi erigentes, und dann durch die Anspannung der Musc. ischio-cavernosi und Musc. transversus perinei profundus und M. bulbo cavernos. eine Verlegung des Venenblutabflusses. Es findet also ein stärkerer Blutzufluß, eine Anhäufung statt bei gleichzeitig gehindertem AbfluB. Bei der Entstehung einer pathologischen Erektion kann es sich also sowohl um einen gesteigerten Zufluß bei gleichbleibendem AbfluB handeln oder um eine venöse Stauung allein durch irgend welche Hindernisse in dem Venenabflußgebiet, schließlich denn auch durch beide Momente zusammen. Das beste Beispiel sind ja Penis se, die künstlich durch erstarrende Injektionsmasse erigiert sind, wie man solche Prachtexemplare in Anatomien bewundern kann. Jede Blutgerinnung in den Schwellkörpern muß demgemäß eine hochgradige Erektion erzeugen, die nicht eher schwindet. bis das Hindernis im Blutabflub durch Erweichung und Resorption beseitigt ist. Wir haben vorhin schon Fälle kennen gelernt, wo die Blutgerinnung durch die veränderte klebrige Beschaffenheit des Blutes bedingt wurde, also ohne Entzündung. Dieselbe Wirkung wird ein intensiver Bluterguß in die Schwellkörper haben, wie dies durch Verletzungen, Zerquetschung oder Schubverletzung (Demarquay und Barlemont) möglich ist. Ein solcher Fall ist auch beschrieben von Vor ster, ${ }^{1}$ ) der einen Bluterguß in das rechte corp. cavern. beobachtete, wo durch die Kompression der ven. profund. penis die Erektion noch gesteigert wurde.

Als weitere Ursache für die Blutgerinnung kennen wir die Entzündung. Entzündet sich durch irgendwelche Einwirkung die Wandung einer Vene, soist die Folge davon die Obliteration des Gefäßes, die Venenthrombose. Ich brauche das des weiteren nicht auszuführen. Bekannt sind die bösen Erkrankungen der Venen der unteren Extremitäten; bekannt ist, wie schmerzhaft eine Phlebitis und Periphlebitis der vena saphena ist, wie lange oft eine solche Erkrankung dauert; bekannt ist, wie gefürchtet die Phlebitis und Thrombose der vena cruralis ist, speziell bei Diabetes melitus.

Wenn wir uns das vergegenwärtigen, so erklärt sich uns sofort das Bild des Priapismus, der durch eine Entzündung der Kavernen, durch eine cavernitis hervorgerufen ist. Rokitansky beschreibt in seinem Lehrbuche einen Fall, in dem

1) Deutsehe Zeitschr. f. Chirurgie. XXVII. 1888. 
bei der Sektion eine Eiteransammlung in den corp. cavernos. als Ursache der noch im Tode fortbestehenden Erektion festgestellt wurde, desgleichen $K$ a uders') eine durch die Sektion festgestellte Thrombosis corp. cavernosorum. Auch Neumann $n^{2}$ beobachtete 2 Fälle von Priapismus infolge Cavernitis.

Die Schmerzhaftigkeit eines solchen Penis ist eine ganz auferordentliche. Der Patient krümmt sich hin und her und kann doch die Rückenlage nicht verlassen, die Beine werden gespreizt gehalten, fast unbeweglich. Geringes Anziehen der Beine oder Ausstrecken steigert schon die Schmerzen, der leiseste Druck der Bettdecke wird nicht vertragen. Qualvoll ist das Urinieren, obschon in meinem Falle an sich keine Schwierigkeiten bestanden. Jedoch jedesmal mußte die Seitenlage dazu eingenommen werden mit Ausnahme der ersten Tage, wo Pat. sich noch freier bewegen konnte und deshalb die Knieellenbogenlage vorzog. Obschon er reichlich Narcotica (Chloralhydrat, Trional mit Morphium, Suppositorien mit Morphium, Cocain, Belladonna) erhielt, brüllte und schrie er zeitweise fürchterlich bis zur Ermattung. Welche Schmerzen ein solcher Patient erdulden $\mathrm{mu} \beta$ (in meinem Falle fast einen Monat), kann nur der ermessen, der aus eigener Anschauung einen solchen Fall beobachtet hat.

Bekannt sind uns ja schon die Klagen unserer GonorrhoeKranken, die von schmerzhaften Erektionen Nachts gepeinigt werden; und dabei handelt es sich nur um eine kurze Spanne Zeit. Diese vorübergehenden schmerzhaften Erektionen bei Entzündung der Urethra, des collum resicae, bei Prostatitis acuta sind bedingt durch den peripheren Nervenreiz auf die nervi erigentes und bilden ein Beispiel für unsere erste Annahme, daß eine länger dauernde Erektion auch nur die Folge sein kann einer gesteigerten arteriellen Blutzufuhr o h n e Behinderung des venösen Abflusses. Jedoch daß auch zentrale Ursachen dieser Art Priapismus erzeugen können, beweisen die Fälle von Verletzung des Rückenmarks nach Fraktur der Wirbelsäule, wie solche wiederholt beobachtet sind, und die DauerErektionen bei Geisteskranken.

Als Ursache des Priapismus ist bei meinem Patienten mit Sicherheit die Gonorrhoe erkannt worden. Durch Einwanderung ron Gonokokken in das cavernöse Gewebe ist eine Cavernitis erzeugt worden, die zur Blutgerinnung und Thrombose führte. Merkwürdig nur ist dabei, daß in den ersten 10 Tagen gar keine Spur von Gonorrhoe zu finden war, weder

1) Wiener med. Jahrbücher 1882.

2) " " " 
Ausfluß noch Flocken oder Trübung im Urin, keine Erkrankung der zugehörigen Drüsenorgane, wie der Cowperschen oder Littréschen Drüsen, oder der Prostata und der Samenblasen. Und doch muß man annehmen, daß der erste Beginn der Erkrankung in einer langsam einsetzenden Entzündung und allmählichen Abszeßbildung in dem periurethralen Gewebe der pars post. urethrae, übergreifend auf die corpora cavernosa penis, gewesen ist, die zu einer Thrombose der venae profund. penis bei ihrem Durchtritt durch den Musc. transversus perinei profundus fübrte, wodurch der Venenblutrückflaß sistiert wurde. So läßt sich auch allein die 2-3 Stunden andauernde schmerzhafte Erektion erklären, die 8 Tage vorher schon einmal bestanden hatte. Damals ist die Passage nochmals gangbar geworden, vielleicht auf kollateralem Wege und durch die vena dorsalis penis. Durch die langsam fortschreitende Arbeit der Gonokokken wurde das Gewebe jedoch allmählich weiter infiltriert, bis vielleicht eine physiologische Erektion dann den Anlaf gab, durch die starke Blutüberfüllung den Durchgang des Venenblutes völlig $\mathrm{zu}$ sperren. Bei den verschiedensten anderen Fällen können die Autoren auch von Vorboten berichten, daß die Patienten, bevor der Hauptanfall kam, schon einmal oder auch schon mehrere Male mit schmerzhaften und stundenweise auftretenden Erektionen zu kämpfen hatten.

Erst am 12. Tage kam es dann zum Durchbruch des Abszesses oder wohl richtiger gesagt, kleinster Abszeßherde in die Urethra, beschleunigt vielleicht durch die nochmalige brüske Untersuchung der hinteren Harnwege per rectum am Tage vorher. Ich sage Abszeßherde, da der Ausfluß, der sich so unerwartet einstellte, nicht sehr reichlich war und nicht so reichlich, wie wir das sonst bei einem Abszeßdurchbruch in die Urethra zu beobachten und von den Patienten zu hören gewohnt sind, hier nur in der Menge einer mäßigen subakuten Gonorxhoe. Und daß der Sitz dieser Erkrankung völlig hinten, in der pars posterior war, zeigte sich daran, daß sich schon am folgenden Tage der Urin, der bis dahin klar gewesen war, trübte und daß sich die Zeichen der Cystitis, spez. vermehrter Harndrang, einstellten.

Charakteristisch für Priapismus ist die Form des erigierten Penis. Die Glans ist nicht geschwellt und vom Präputium bedeckt. Die Thrombose erstreckt sich offenbar nicht auf das cavernöse Gewebe der Glans, das bekanntlich die Fortsetzung des corp. cavern. urethrae ist. Hier muß ein völliger Verschluß des Venenblutabflusses nicht möglich sein, es sind wohl Anastomosen mit den Venen der Haut des Penis vorhanden, die in meinem 
Falle auch stets stark gefüllt waren, wie es ebenfalls auf der Photographie deutlich sichtbar ist. Wäre ein völliger Verschluß des Venenblutes im ganzen Penis vorhanden, so müßte es ja auch unbedingt zur Gangrän des Penis kommen. Aber auch die Venen des corpus cavern. urethrae müssen in meinem Falle durchgängig gewesen sein, sie konnten nicht phlebitisch erkrankt sein, weil sonst die Urinentieerung mehr erschwert gewesen wäre. Bei der physiologischen Erektion, bei der alle drei Schwellkörper strotzend gefüllt sind, ist bekanntlich eine Miktion nicht möglich, während bei Priapismus die Urinentleerung in einzelnen Fällen wohl erschwert, meist jecioch unbehindert war. Die Urinentleerung ist höchstens dadurch erschwert, daß die Patienten gezwungen sind, aus der Rückenlage in die Seitenlage oder Knieellenbogenlage übergehen zu müssen, um sich nicht zu beschmutzen.

Fernerhin charakteristisch bei Priapismus ist die Neigung des erigierten Penis stark zum Nabel aufwärts, meist bis fast an ihn heranreichend. Der Winkel zwischen Penis und Bauchdecken beträgt $45^{\circ}$ oder weniger. Dabei ist eine Verschiebung des Penis nach oben oder unten, nach rechts oder links völlig unmöglich. Die physiologische Erektion gewährt immerhin eine' gewissen Spielraum in der Beweglichkeit, bei Priapismus steht der Penis an seiner Wurzel so fest wie angegossen. Pollutionen erfolgen dabei nie.

Die erste Frage, die der unglückliche Besitzer einer solchen Dauer-Erektion an den untersuchenden Arzt richtet, ist die, wie lange wird der qualrolle Zustand noch andauern. Und da ist es sehr schwer, eine tröstende Antwort zu geben. Denn die meisten beobachteten Fälle waren nicht unter 14 Tagen, meist länger, bis zu 2 Monaten. Und wenn man sich über das Wesen der Erkrankung klar ist, die eben meistens in der Thrombose des cavernösen Gewebes besteht, so ist das ohne weiteres verständlich.

Die Therapie weicht dabei kaum, soweit es sich um Thrombose bei Priapismus handelt, von der gewöhnlichen ab, wie wir sie bei Phlebitis und Thrombose der Venen der unteren Extremität anzuwenden gewohnt sind. Im Anfange haben wir Kälte anzuwenden in Form von kühlenden Umschlägen oder Eisbeutel, resp. Kühlschlangen von Aluminium, um weiterer Entziindung vorzubeugen. Dann gehen wir, um Resorption zu erreichen, zur Behandlung mit Wärme über. Wir nehmen dazu Priessnitzsche Umschläge, entweder mit gewöhnlichem warmem Wasser oder mit essigsaurer Tonerde 
oder Kamillenaufguß; ferner Breiumschläge von Leinmehl mit oder ohne Zusatz von Narcoticis, spez. Kampfer, oder lassen durch die um den Penis gelegten Aluminiumschlangen warmes Wasser zirkulieren. Vor allem wirkten jedoch günstig in unserem Falle die protahierten warmen Bäder, die bis zu 4 Stunden Dauer gegeben wurden. Dazu kann man dann, falls die Schmerzhaftigkeit es zuläßt, den Penis mit Jodsalben, Jodrasogen, mit Ichthyolsalben oder narkotischen Salben wie Kompositionen mit Hyoscyamus und Belladonna einreiben. Blutegel an dem Penis selbst oder ans Perineum gesetzt wirken schmerzlindernd, sind jedoch ohne Erfolg auf die Beseitigung der Erektion an sich.

Ebensowenig konnte ich mir etwas versprechen von Inzisionen in die corp. cavernosa, was auch von einzelnen Beobachtern empfohlen wurde. Würden wir vielleicht in eine Thrombose der vena cruralis oder saphena einschneiden? Ich glaube kaum. Rose ${ }^{1}$ ) machte zwar die Inzision eines Hämatoms, das Priapismus durch Blutergub in das rechte corp. cavern. hervorgerufen hatte, mit dem gewünschten Erfolge, und ebenso bei einem 23jährigen Bluter Circumcision, um durch Blutentziehung eine Erschlaffung des Penis herbeizuführen, was ihm auch gelang, indem nach 3stündiger Blutung am 4. Tage nach der Operation der Priapismus fiel, der bereits 36 Tage angedauert hatte.

Intern muß man vor allem reichlichen. Gebrauch von Nircoticis machen und erreichten wir am meisten mit Trional $1 \cdot 0+$ Morph. 0.015 und mit Anwendung von Suppositorien von Cocain, Morph. und Belladonna. Von anderen (W in d is h, Schultze) wird als besonders wirksam empfohlen konstant Opium 0.04 mit Kampfer 0.1 zu geben. Matthias glaubt einen Erfolg der 14tägigen Anwendung von Sol. Kol. jodat $5 \cdot 0: 150 \cdot 03 \times$ tgl. 1 EBlöffel zuschreiben zu dürfen, während Rosenthal ${ }^{2}$ ) gute Wirkung von Tinct. Veratri virid. (3stündl. 10 Tropfen) sah.

Daß außerdem die causa morbi, in meinem Falle die Gonorrhoe, bekämpft werden muß, ist selbstverständlich. Die dabei angewandten Mittel habe ich oben schon angeführt.

1) Deutsche Zeitschr. f. Chir. XXVI. 1888.

2) Anzeiger der k. k. Gesellschaft der Ärzte in Wien. 1882. N. 19. 
Mehr oder weniger vergeht jedoch trotz der besten Mittel und trotz der größten Mühe, die man sich gibt, eine längere Zeit, bis der Penis wieder erschlaftt. Und noch ehe es so weit ist, legt sich Arzt und Patient die Frage vor, wie wird es in Zukunft sein, wird die Erschlaffung dann eine dauernde sein oder wird die physiologische Erektion sich wieder einstellen. In 2 Fällen ist dauernde Impotenz (Matthias und Salzer) die Folge gewesen, was jedoch wohl durch das Allgemeinleiden, die Leukämie, bedingt gewesen sein wird, der die Patienten innerhalb eines Jahres erlagen. In den meisten Fällen scheint die Potentia coeundi nicht verloren zu gehen, so auch in meinem Falle - zur größten Freude meines Patienten. 